\title{
Genital Tuberculosis - An Uncommon Presentation
}

\author{
N AKHTER ${ }^{\mathrm{a}}$, A KHANAM ${ }^{\mathrm{b}}$, F BEGUM ${ }^{\mathrm{c}}$
}

\section{Summary:}

A married women of 30 years, mother of one child, housewife was referred to out patient department of Khulna Medical College Hospital with history of blood stained vaginal discharge, secondary amenorrhoea for 3 years and evening rise of temperature and anorexia for 3 months. On speculum examination, cervix was oedematous, bright red in colour with papillary growth which bleeds on touch. She also had bilateral excavated lesion at the lowest part of the vagina close to the introitus which was red in colour with undermined edge. Visual inspection aided by acetic acid (VIA) was positive. Colposcopy guided biopsy was taken from unhealthy areas. There was extensive mottling on chest $X$-ary. She had high ESR, AFB+ve on sputum culture. The patient was diagnosed as a case of active pulmonary

\section{Introduction:}

Female genital TB is not uncommon in countries where pulmonary TB is wide spread. But tuberculosis of vulva and vagina is very rare and it is seen only in $1-2 \%$ of genital tract TB. Tuberculosis of cervix accounts for 0.1 to $0.65 \%$ of all cases of TB and $5-24 \%$ of genital tract TB $1,2,3,4,5,6,7,8$. In $92 \%$ of cases, genital TB is secondary to focus in the lungs, lymph nodes urinary tract, bones and joints. Genital organs most frequently affected include fallopian tubes (95-100\%), endometrium (50-60\%), and ovaries (20-30\%). Mostly genital tuberculosis is diagnosed during evaluation for infertility. Major presenting symptoms of genital TB are infertility (45-55\%) pelvic pain (50\%), poor general health (25\%) and menstrual disturbance (20\%). Female genital tuberculosis is treated with the same combined

a. Dr. Nasreen Akhter, FCPS, Assistant Professor, Obs \& Gynae, Khulna Medical College, Khulna.

b. Dr. Afroza Khanam, DGO, Assistant Professor, Obs \& Gynae, Khulna Medical College, Khulna.

c. Dr. Fouzia Begum, MCPS, Junior Consultant, Obs \& Gynae, Khulna Medical College Hospital, Khulna.

Address of Correspondence: Dr. Nasreen Akhter, FCPS, Assistant Professor, Obs \& Gynae, Khulna Medical College, Khulna, Mobile: 01712248062, Email: drnasreenkabir@gmail.com

Received: 29 May, 2011

Accepted: 13 March, 2012
TB. Histopathological report of cervical tissue showed granulomatous lesion. Patient was given a regimen of standard anti TB drugs. After 2 weeks, during her first follow up, patient had few symptoms with regression of cervical growth and disappearance of vaginal ulcer. Patient herself stated about her wellbeing after the start of antiTB drugs.

Though cervical TB is not uncommon among genital TB (5-24\%), vaginal tubercular lesion is very uncommon and concurrent pulmonary, endometrial, cervical and vaginal tuberculosis is a rare event. Careful evaluation is needed to diagnose tubercular infection in genital organs specially in GOPDs and colposcopy clinics.

(J Bangladesh Coll Phys Surg 2012; 30: 108-111)

drug therapy used in pulmonary and extrapulmonary tuberculosis but the diagnosis is critical. This paper briefly describes the concurrent occurrence of pulmonary endometrial, cervical and vaginal tuberculosis.

\section{Case History:}

A 30 years old lady, married for 5 years, Para-1, ALC3 yrs, housewife was referred to out patient department of Khulna Medical College Hospital (KMCH) by a general practitioner. The patient gave history of excessive blood stained, pervaginal discharge, chronic pelvic pain and amenorrhoea for 3 years since her last childbirth. She was not taking any contraceptive. She complained of evening rise of temperature and anorexia for 3 months. The patient was admitted in gynae ward for thorough evaluation. Patient gave no family history of pulmonary TB. On examination, patient was found ill looking and anxious. Her Temperature was $99^{\circ} \mathrm{F}$ and she was normotensive. Pervaginal examination revealed normal sized uterus, bilateral excavated lesion size $2 \mathrm{~cm}$ $\times 2 \mathrm{~cm}$ with irregular outline and undermined edge present in the lowest part of vagina close to the introitus (Fig-III \& IV). Cervix was oedematous with papillary growth almost entirely covering the ectocervix which bled on touch (Fig-I). 


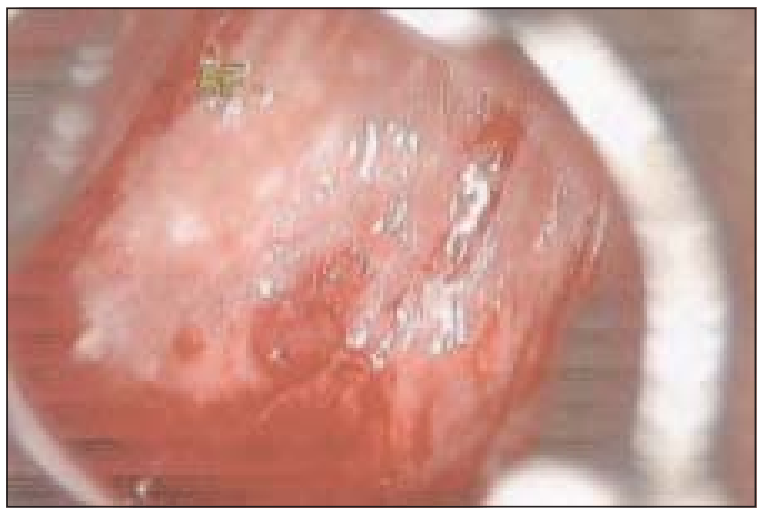

Fig.-1: Colposcopic feature of cervix-Oedematous soft cervix with papillary growth covering the ectocervix bleeds on touch.

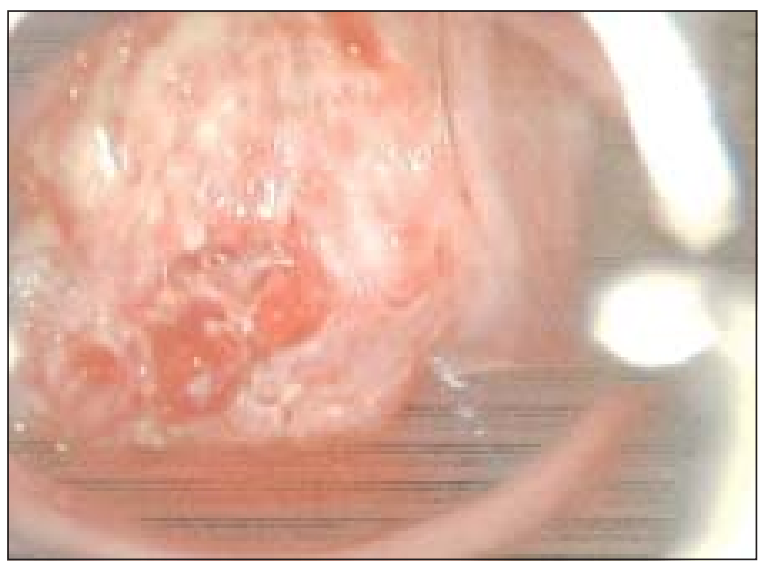

Fig.-2: Cervical colposcopic features after 2 weeks of anti TB treatment showing much regression of growth with small congested area.
VDRL, TPHA, HIV Elisa test \& HVS with gram staining and culture was done to exclude syphilis, gonococcal, trichomonal and HIV infection. The patient had high ESR (125mm/hrs) polymorphonuclear leukocytosis with lymphopenia, milliary mottling on chest $\mathrm{X}$-ray, sinus tachycardia on ECG and AFB+ve on sputum culture. Paps cytology reveled inflammatory cells with mild dyskaryosis. Urine samples were negative for AFB. Ultrasonogram of whole abdomen showed no abnormality. Endometrial aspiration cytology and Mantonx test were not done due to presence of active tuberculr lesion. Cervical biopsy report revealed granulomatous lesion with plenty of mononuclear cells and caseation. The patient was referred to the department of medicine of KMCH seeking opinion. The case was diagnosed as bilateral extensive pulmonary TB with concomitant endometrial, cervical and vaginal TB. The patient was treated with anti TB drugs (INH+ Rifampicin+Ethambutol+ Pyrazinamide). After One week of initiation of treatment, Ethambutol was omitted as the patient complained of visual disturbance. After 2 weeks of onset of treatment during follow up, the patient had better look with no P/V discharge and no ulcerative lesion in cervix (Fig-II) and vaginal ulcer was totally disappeared (Fig-V). Patient is still on antitubercular drugs and on regular follow up.

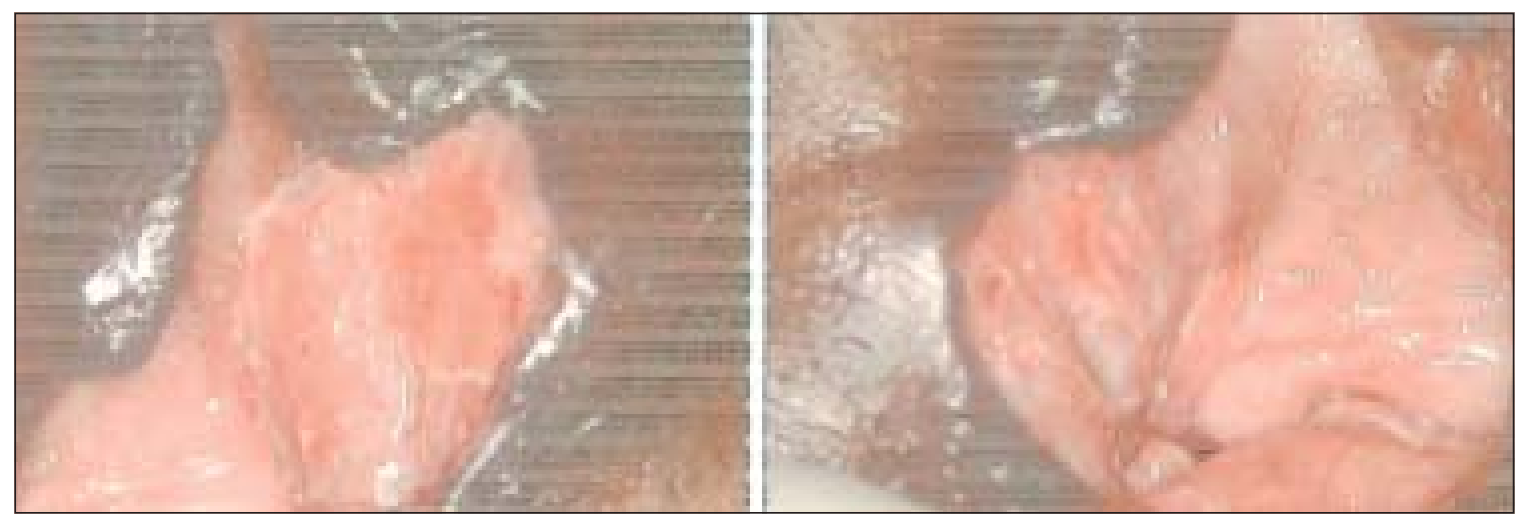

Fig.-3\&4: Colposcopic feature of vagina- Bilateral vaginal ulcer with undermined edge. 


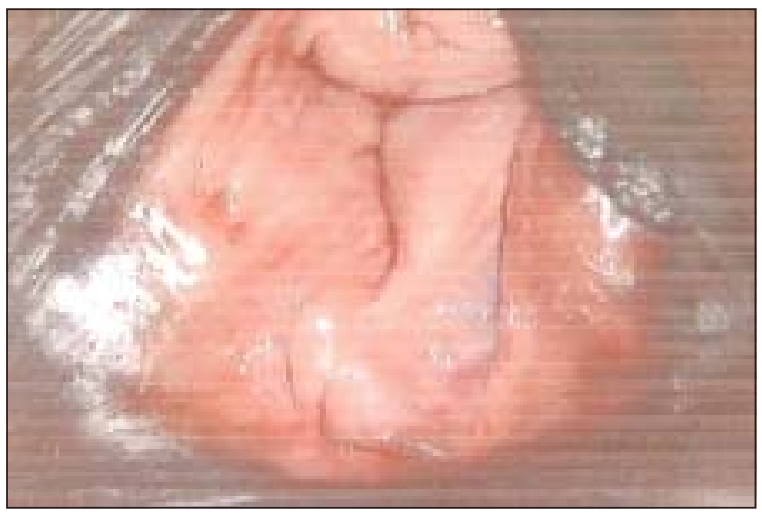

Fig.-5: Colposcopic feature of lower part of vagina showing no ulceration after 2 weeks of anti TB treatment.

\section{Discussion:}

Tuberculosis is one of the oldest disease known to affect human ${ }^{15}$. Female genital TB is a rare disease in some developed countries but it is a frequent cause of chronic pelvic inflammatory disease (PID) and infertility in other parts of the world ${ }^{16}$. Symptomatic genital tract TB usually present with abnormal vaginal bleeding, menstrual irregularities, amenorrhoea, abdominal pain \& constitutional symptoms $s^{5,6,9,10,11}$.

Pelvic organs are infected from a primary focus, usually the chest, by haematogenous spread 2, 4,5, 10,12. The cervix is infected as a part of this process, by lymphatic spread or by direct extension. The vagina and vulva are rarely involved. The primary lesion is often healed by the time of presentation $5,6,7,8,9,10,11,12,13$. Some authors suggest the existence of primary genital tuberculosis which may spread by venereal transmission. These lesions are extremely rare and usually present as isolated chronic ulcerative lesions of the external genitalia in absence of TB of the upper urogenital system ${ }^{17}$. The presence of extra genital foci of $\mathrm{TB}$, as a rule is rare when genital lesion is discovered. The extent of genital lesion may be minimal or advanced. Minimal genital TB is usually asymptomatic except for sterility. Examination of pelvis may reveal no abnormality. In rare cases, cervical $\mathrm{TB}$ may be a primary infection ${ }^{2,4,5,10,13}$ introduced by a partner with tuberculous epididymitis or other genitourinary disease. It is uncommon for tuberculosis to involve the vulva and vagina. The gross appearance may be ulcerative with multiple sinuses, it may be hypertrophic with elephantiasis, or it may be similar to that of carcinoma.
There may be hormone dependence of infection 2,5 given that $80 \%$ of cases occur in the reproductive age.

The macroscopic finding of cervical and vaginal TB were illustrated by this case. There may be papillary or vegetative growth, a milliary appearance or ulceration present thus simulating invasive cervical cancer.

Microscopically, there are caseating granulomata in cervical lesion. The differential diagnosis of granulomatous disease of cervix include amoebiasis, schistosomiasis, brucellosis, sarcoidosis and foreign body reaction. The diagnosis of the cervical and vulvovaginal TB is usually made by histological examination ${ }^{3,9,12}$ of cervical and vulvovaginal biopsy. Specimen staining of AFB is not found to be very useful in making the diagnosis of genital $\mathrm{TB}^{14}$. Cervical cytology showed inflammatory cells with mild dyskaryosis therefore, the presence of typical granuloma is sufficient for diagnosis if other causes of cervicitis are excluded or primary focus identified. In this case the primary focus in thought to be lung which is identified and other causes of the ulcerative cervical lesion e.g. syphilis, malignancy is excluded. Molecular probe may be more sensitive than culture but also have reduced specificity.

Khilani and colleagues reported that when the cervical cytologic smear reveals the presence of clusters of epitheloid cells, it may be suggestive of tuberculous lesion of cervix, but it would be diagnosed histologically and /or bacteriologically.

Once the diagnosis of genital TB is confirmed, it is important to rule out TB in other parts of the body. A chest radiograph and three early morning sputum or gastric aspirate samples or early morning urine samples for AFB stain and culture and IVU are recommended ${ }^{19}$.

Daly and Monif reported that $10 \%$ of females with genital TB also show evidence of renal TB. Here in this case, endometrial, cervical and vaginal infection exists concurrently with the active pulmonary infection which is theoretically primary. Endometrial sampling and biopsy of endometrium and vaginal lesion was posponded because of presence of active pulmonary lesion evidenced by AFB in sputum, extensive pulmonary mottling in chest $\mathrm{X}$-ray and high ESR. After completion of TB treatment endometrial and vaginal sampling should be done for biopsy irrespective of resumption of menstruation. But there is strong 
possibility of endometrial involvement as the patient suffered from amenorrhoea for last three years though biopsy could not have been done.

Before the advent of effective chemotherapy, surgery was the mainstay of treatment of genital tract TB and post operative complication such as bowel fistula (14\%) and mortality from primary disease (2.2\%) were high. Experts suggests that extrapulmonary TB may be even easier to treat than pulmonary TB owing to the decreased concentration of organisms in these lesions and increased accessibility of the sites. If surgical intervention is needed, chemotherapy makes it safer, easier and more effective if the regimen contain multiple drugs and taken regularly for a sufficient period of time. In the index case, though standard anittubercular regimen was initiated, Ethambutol was withdrawn due to visual problem of the patient as optic neuritis is a recognized side effect of Ethambutol in some cases ${ }^{20}$. The major determinant of the outcome of treatment is the patient adherence to the drug regimen.

A lesion on the genital tract provides a marker to assess response to therapy. Here in this case, only after two weeks, vaginal ulcer completely disappeared and as such biopsy could not be done. Cervical growth also disappeared except a small area of congestion. The lesion should respond to six months standard antitubercular therapy but in the index case the response was dramatic.

Histological examination of serial biopsy can confirm therapeutic response Complete hemogram should be done with sputum culture for AFB after six weeks. Radiological follow up should be done by USG and MRI in presence of amenorrhoea. MRI is better to visualise endometrium, myometrium and junctional zone and any appearance consistent with Asherman's syndrome and lymphadenopathy. Fertility is very poor even after treatment owing to endometrial and tubal involvement at presentation and subsequent healing by fibrosis ${ }^{10,11.12}$. The incidence of TB has increased recently and is partly attributable to HIV pandemic. There should be high index of suspicion of genital tuberculosis in women with abnormal appearance of cervix, vulva and vagina in area where HIV and TB is prevalent.

\section{References:}

1. Carter JR. Unsual presentations of genital tract tubeculosis. Int J Gynaecol Obstet 1990;33:171-6.

2. Carter J, Peat B, Dalrymple C,et al. Cervical tuberculosiscase report. Aust NZ J Obstet Gynaecol.1989;29:270-1.

3. Koller AB. Granulomatous lesions of the cervix uteri in black patients. South Afr Med J. 1975;49:1228-32.

4. Richards MJ, Angus D. Possible sexual transmission of genitourinary tuberculosis. Int J TB Lung Dis.1998;2:439.

5. Chowdhury NNR. Overview of tuberculosis of the female genital tracty. J Indian Med Assoc. 1996;94:354-61.

6. Kobayashi-Kawata T. Tuberculous cervicitis. Acta Cytol 1978;22:193-4.

7. Chakraborty P, Roy A, Bhattacharya S, et al. Tubeculous cervicitis: a clinicopathologyical and bacteriological study. J Indian Med Assoc. 1995;93:167-8.

8. Nogales-Ortiz F, Tarancon I, Nogales FF Jr. The pathology of female genital tuberculosis. A 31 uear study of cases. Obstet Gynecol. 1979;53;4229.

9. Shobin D, Sall S, Pellman C. genitourinary tuberculosis simulating cervical carcinoma. J Reprod Med 1976;17:305-8.

10. Sinha R, gupta D, Tuli n. Genital tract tuberculosis with myometrial involvment.Int J Gynaecol Obstet. 1997;557;191-2.

11. Highaman WJ. Cervical smears in tuberculous endometritis. Acta Cytol 1972;16:16-20.

12. Sutherland Am, Glen Es, macfarlane JR. Transmission of genito-urinary tuberculosis. Health Bull 1982; 40: 87-91.

13. Bhambani S, das DK, Singh V, et al. Cervical tuberculosis with carcinoma in situ:a cytodiagnosis. Acta Cytol 1985; 29: $87-8$.

14. Agarwal J. Female genital tuberculosis- a retrospective clinicopathologic study of 501 cases. Indian J Pathol Microbiol 1993;36:383-97.

15. Raviglione MC, O,Brien RJ. Tuberculosis. In: Fauci AS, Brauwal E, Isselbacher KJ, Wilson JD, Martin JB, Kasper DL et al., editors. Harrisons, principles of internal medicine. New york: MacGraw-Hill, 2001:1024-1035.

16. Martens MG. Pelvic inflammatory disease. In: Rock JA, Thompson JD, Lippincot- Raven,1997:678-685.

17. Miller JW. Vulvar tuberculosis. Tubercle J, 1979;17:3-6.

18. F. Akhlaghi \& A. Hamedi : Postmenopausal Tuberculosis of The Cervix, Vagina And Vulva. The Internet Journal of Gynecology and Obstetrics. 2004 Volume 3 Number 1

19. Varma T, Glob Libr, Women’s med, (ISSN 1756-2228) 2008, DOI 10.3843/GLOWM.10034.

20. http://stason.org/TULARC/health/Drugs-Herbs-Manual/ Ethambutol.html. 